\title{
LAUFER (Jacqueline), MARRY (Catherine), MARUANI (Margaret). - Le Travail du genre: Les sciences sociales à l'épreuve des différences de sexe
} Paris : La Découverte, 2003. - 368 p.

Rebecca Rogers

\section{OpenEdition}

Journals

Édition électronique

URL : https://journals.openedition.org/histoire-education/1163

DOI : 10.4000/histoire-education. 1163

ISSN : 2102-5452

Éditeur

ENS Éditions

Édition imprimée

Date de publication : 1 janvier 2005

Pagination : 138-139

ISBN : 2-7342-1006-1

ISSN : 0221-6280

\section{Référence électronique}

Rebecca Rogers, «LAUFER (Jacqueline), MARRY (Catherine), MARUANI (Margaret). - Le Travail du genre : Les sciences sociales à l'épreuve des différences de sexe », Histoire de l'éducation [En ligne], 105 | 2005, mis en ligne le 23 mars 2009, consulté le 20 mai 2021. URL : http://journals.openedition.org/ histoire-education/1163; DOI : https://doi.org/10.4000/histoire-education.1163

Ce document a été généré automatiquement le 20 mai 2021.

(C) Tous droits réservés 
LAUFER (Jacqueline), MARRY (Catherine), MARUANI (Margaret). Le Travail du genre : Les sciences sociales à l'épreuve des différences de sexe

Paris : La Découverte, 2003. - 368 p.

\author{
Rebecca Rogers
}

\title{
RÉFÉRENCE
}

LAUFER (Jacqueline), MARRY (Catherine), MARUANI (Margaret). - Le Travail du genre : Les sciences sociales à l'épreuve des différences de sexe. - Paris : La Découverte, 2003. - 368 p.

1 Ce volume est le fruit d'un colloque organisé en 2002 par le groupement de recherche MAGE (Marché du travail et genre). L'objectif était d'observer l'émergence d'analyses sexuées dans le monde du travail, et cela à partir de différents points de vue disciplinaires : ceux de l'histoire, de la sociologie, des sciences économiques, de la statistique et de la démographie, enfin, du droit et des sciences politiques. Pour les lecteurs d'Histoire de l'éducation, l'intérêt du volume est double. Il témoigne de l'essor de recherches sur les femmes et le genre et des «vertus heuristiques d'une lecture sexuée du monde social» (9); par ailleurs, plusieurs articles de chercheurs en sociologie de l'éducation évoquent spécifiquement le domaine de l'éducation. Celui-ci est abordé comme un des facteurs clés à prendre en compte pour comprendre le genre du travail de nos jours.

2 Les auteurs relatent l'émergence des approches sexuées dans l'étude du système scolaire pour montrer ensuite comment l'approche du genre change notre compréhension. Pour Roger Establet et Marlaine Cacouault-Bitaud, traitant de la 
France, les approches sexuées sont fort récentes par rapport à la sociologie du travail ; il faut attendre les années 1990 avec les livres de Marie Duru-Bellat, L'École des filles (1990) et Christian Baudelot et Roger Establet, Allez les filles ! (1992) pour que les chercheurs s'intéressent au paradoxe de la meilleure réussite scolaire des filles et de leur orientation vers des filières les moins «rentables ». Malgré de telles études et les recherches de M. Cacouault-Bitaud, l'article qu'elle consacre au sexe des enseignants montre que cette question peut ne susciter aucun questionnement dans les travaux les plus récents, en dépit de la féminisation importante des métiers de l'enseignement. R. Establet se penche sur ceux qui ont suivi cette voie de recherche et distingue trois interprétations sociologiques sur la réussite des filles à l'école, suivant l'analyse de Catherine Marry. Dans les trois cas, il montre qu'il faut sortir de l'école pour la comprendre. Et c'est encore mieux quand on la compare à d'autres systèmes scolaires, comme le propose Juliana Jacobi dans un article très informatif sur l'état des recherches sur le genre dans les sciences de l'éducation en Allemagne. À travers une étude du Zeitschrift für Pädagogik et les rapports du groupe de travail du Max Planck Institut depuis 1979-1980, elle brosse un panorama de la recherche en Allemagne; celle-ci est bien différente de celle qui se développe outre-Rhin, elle focalise notamment son attention sur la question de la mixité dans la réussite différentielle des sexes. Pourtant, elle conclut, comme la majorité des auteurs du volume, que les recherches féministes et celles plus "classiques ", ici en sociologie de l'éducation, se croisent assez peu. La reconnaissance du domaine de recherche est encore loin d'avoir obtenu la reconnaissance de l'institution, notamment en Allemagne où les femmes participent bien moins qu'ailleurs en Europe à l'enseignement supérieur et secondaire.

\section{AUTEURS}

REBECCA ROGERS 